\title{
Gastrobronchial Fistula as a Complication of Bariatric Surgery: A Series of 6 Cases
}

\author{
Nasser Sakran ${ }^{a} \quad$ Ahmad Assalia $^{b}$ Andrei Keidar ${ }^{c}$ David Goitein ${ }^{d, e}$ \\ a Department of Surgery A, Hillel Yaffe Medical Center, Hadera, ${ }^{b}$ Department of Surgery, \\ Rambam Health Care Campus, Haifa, 'Department of Surgery, Hadassah-Hebrew University \\ Medical Center, Jerusalem, dDepartment of Surgery C, Chaim Sheba Medical Center, \\ Tel Hashomer, ${ }^{e}$ Sackler School of Medicine, Tel Aviv University, Tel Aviv, Israel
}

\section{Key Words}

Morbid obesity • Laparoscopic sleeve gastrectomy • Roux en Y gastric bypass • Gastric leak • Gastrobronchial fistula

\begin{abstract}
Objective: To present a multicenter series of 6 patients who developed gastrobronchial fistula (GBF). GBF is a rare subtype of gastric leaks following bariatric surgery, which is the mainstay of treatment for the obesity pandemic. Methods: We retrospectively identified 6 patients with GBF (out of 2,308 cases performed: $0.2 \%$ ). One patient had undergone Roux-en-Y gastric bypass, and 5 had a sleeve gastrectomy. Demographics, previous surgeries, clinical presentation, timing of fistula diagnosis, diagnostic and treatment measures employed, and outcome were collected. Results: Four patients were female, the average age and BMI were 42 years and $42.5 \mathrm{~kg} / \mathrm{m}^{2}$, respectively. Three patients had previous surgeries (Nissen fundoplication, adjustable gastric banding, and vertical banded gastroplasty). Median time to fistula diagnosis was 40 days (range 15-90 days). Clinical presentation included chronic cough, hemoptysis, dyspnea and fever as well as persistent left pleural effusion or pneumonia. Diagnosis was confirmed by computed tomography in all cases. Two patients were treated nonoperatively, while 4 eventually required surgery for resolution. Left lower lobectomy was necessary in 3 of 4 cases. Concomitant procedures were total gastrectomy in 2 cases and conversion of a sleeve to a gastric bypass in 1 case. Resolution occurred 30 days to 2 years after initial surgery. No mortalities were encountered. Conclusions: GBF is a rare but devastating complication following bariatric surgery. It may develop as a late complication of a chronic upper gastric leak. Surgery is curative although nonoperative management may be warranted in selected cases.
\end{abstract}


Sakran et al.: Gastrobronchial Fistula as a Complication of Bariatric Surgery: A Series of 6 Cases

\section{Introduction}

Laparoscopic Roux en Y gastric bypass (LRYGB) and sleeve gastrectomy (LSG) are performed routinely for treatment of morbid obesity [1-3]. One of the dreaded complications after these procedures is gastric leak. This most commonly occurs at the upper stapler line, near the gastroesophageal junction in LSG, and at the gastrojejunostomy site in LRYGB $[4,5]$. This complication, if not identified and treated quickly and aggressively, may lead to abdominal sepsis, occasionally progressing to multiorgan failure and death.

There is no consensus regarding the precautions to be taken during surgery to detect or prevent this complication. Intraoperative endoscopy, air-leak test and transgastric dye injection have been utilized by some authors to detect a leak during initial surgery or when returning after a suspected or proven leak [6-10]. Gastric leak is not uncommon, occurring in $0.1-5.4 \%$ of LRYGB and LSG [4, 11-14]. A minute subset of these will evolve into gastrobronchial fistulae (GBF).

GBF is a rare complication after gastric and esophageal surgery. It has been sporadically reported following surgery for treatment of malignant and benign etiologies such as esophagectomy for carcinoma of the esophagus and fundoplication for reflux [15-18]. Anecdotal cases have also been reported after occurrence of subphrenic abscess, with diaphragmatic erosion following splenectomy or trauma [19].

GBF after bariatric surgery is infrequent. There have been some case reports after gastric band placement, LSG, and LRYGB [20-23].

The clinical presentation suggesting this complication includes persistent cough, sometimes associated with liquid or food ingestion, hemoptysis, wheezing, pleuritic pain, and findings consistent with left pleural effusion or lung consolidation. Fever, leukocytosis, hypoxemia, or frank sepsis may be present.

Diagnostic measures utilized to verify the presence of GBF are contrast swallow studies, computed tomography (CT), endoscopy, and bronchoscopy.

We herein report of 6 cases of GBF after LSG and LRYGB. Clinical presentation, diagnostic efforts and management are elaborated.

\section{Patients and Methods}

Four bariatric centers in Israel participated in this study, each maintaining a prospectively collected database of all bariatric procedures performed. Institutional review board approval for database management was obtained at each center. Retrospective analysis of these databases was carried out querying all LRYGB and LSG cases performed between June 2006 and May 2011 for patients who developed GBF. Other data collected included patient demographics, anthropometrics, operative and perioperative data, and the presence or absence of leaks. Methods used for GBF detection and management as well as time intervals between surgery and diagnosis and between surgery and fistula resolution were recorded.

Surgical technique varied slightly between centers in respect to various parameters including: stapler manufacturer choice, staple height, bougie size, utilization of buttressing materials and/or oversuturing of staple line and choice of energy source (ultrasonic scalpel vs. Liga-sure ${ }^{\mathrm{TM}}$ device). The greater curvature of the stomach was freed prior to vertical division alongside a bougie in all LSG cases.

\section{Results}

Between June 2006 to May 2011, 1,102 patients underwent LRYGB, and 1,206 patients underwent LSG for treatment of morbid obesity in four bariatric centers. All patients met the accepted NIH criteria for bariatric surgery and underwent a thorough preoperative eval- 
Table 1. Patient characteristics

\begin{tabular}{|c|c|c|c|c|c|c|}
\hline Patient number & Age, years & Gender & $\mathrm{BMI}, \mathrm{kg} / \mathrm{m}^{2}$ & Comorbidities & Previous surgery & Procedure \\
\hline 1 & 59 & female & 44 & Chol., OSA & - & LSG \\
\hline 2 & 23 & male & 45 & - & - & LSG \\
\hline 3 & 40 & female & 40 & DM & LAGB & LSG \\
\hline 4 & 57 & female & 45 & HTN & SRVG & LSG \\
\hline 5 & 46 & female & 39 & DM, OSA & Nissen fundoplication & LRYGB \\
\hline 6 & 29 & male & 42 & - & - & LSG \\
\hline
\end{tabular}

Chol = Hypercholesterolemia; OSA = obstructive sleep apnea; DM = diabetes mellitus; HTN = hypertension; LAGB = laparoscopic adjustable gastric banding; SRVG = silastic ring vertical gastroplasty; LSG = laparoscopic sleeve gastrectomy; LRYGB = laparoscopic Roux-en-Y gastric bypass.

uation. We identified $14(1.3 \%)$ and 20 patients (1.6\%), who were diagnosed with gastric leaks after LRYGB and LSG, respectively (1.47\% overall). Six of these developed GBF (17\% of leaks; $0.2 \%$ of all cases).

The average age and BMI were 42 years and $42.5 \mathrm{~kg} / \mathrm{m}^{2}$, respectively. Four of the 6 patients were female.

Comorbid conditions were present in 4 patients (66\%). These included hypertension (n $=1)$, dyslipidemia $(n=2)$, obstructive sleep apnea $(n=2)$, and type 2 diabetes $(n=1)$. Patients with obstructive sleep apnea did not use positive airway pressure devices in the postoperative period. Three patients had previous gastric surgeries (Nissen fundoplication, adjustable gastric banding, and vertical banded gastroplasty). An intraoperative leak test by blue dye injection was performed in all patients. Table 1 summarizes patient characteristics.

Postoperative swallow studies were performed, but were uninformative, in 4 of 6 patients.

GBF presentation differed among the 6 patients and can be divided into three groups according to timing and clinical setup:

Three patients: Immediate postoperative leak (2-3 days) leading to chronic leak with multiple drainage attempts, eventually developing left diaphragm erosion and GBF formation.

Two patients: Para-sleeve fluid collections (infected hematomas) 3-4 weeks after surgery treated by percutaneous, CT-guided drainage, later developing left diaphragm erosion and GBF formation.

One patient: Pulmonary complaints beginning 5 months postoperatively, namely incessant cough, low-grade fever and expectoration of surgical endoclips.

Chronic cough and physical examination compatible with left lower lung consolidation was present in all patients at the time of GBF diagnosis, and raised the suspicion of this entity. Diagnostic modalities utilized were radiographic and endoscopic as follows:

Plain upper abdominal and chest films performed for evaluation of chronic cough showed migration of an intra-abdominal drain into the pleural cavity in 1 patient (fig. 1). Follow-up CT scan demonstrated a communicating tract of air from the gastric sleeve to a bronchus (fig. 2). Four of the patients with suspected leak or GBF underwent water-soluble contrast swallow, which demonstrated a fistula in 2 cases (fig. 3). CT scans were used to evaluate all patients at some point in their course and showed findings compatible with GBF in 4 cases (fig. 1).

Gastroscopic evaluation was performed in 3 patients and identified a fistulous opening in 2 patients. One patient underwent bronchoscopy which was negative. 
Sakran et al.: Gastrobronchial Fistula as a Complication of Bariatric Surgery: A Series of 6 Cases

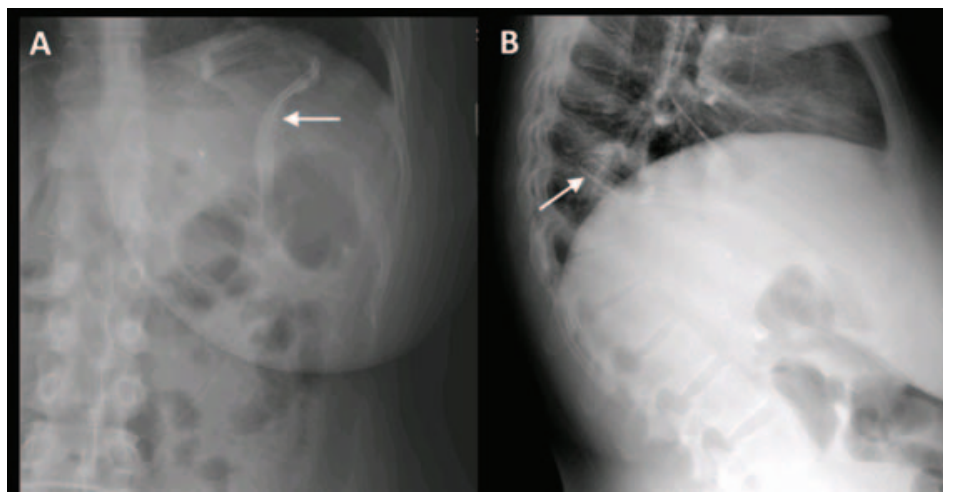

Fig. 1. A Plain abdominal x-ray (antero-posterior view) in a patient with a chronic leak following laparoscopic sleeve gastrectomy. A drain (arrow) is seen curled under the diaphragm. The patient complained of left shoulder pain, attributed to irritation by a subdiaphragmatic abscess (and perhaps the drain itself). No respiratory symptoms were present at this time. B Lateral chest X-ray taken 2 weeks later, prompted by chronic cough onset and clinical signs of left lower lobe (LLL) consolidation. Drain (arrow) can be seen traversing the diaphragm, within the (LLL) consolidation.

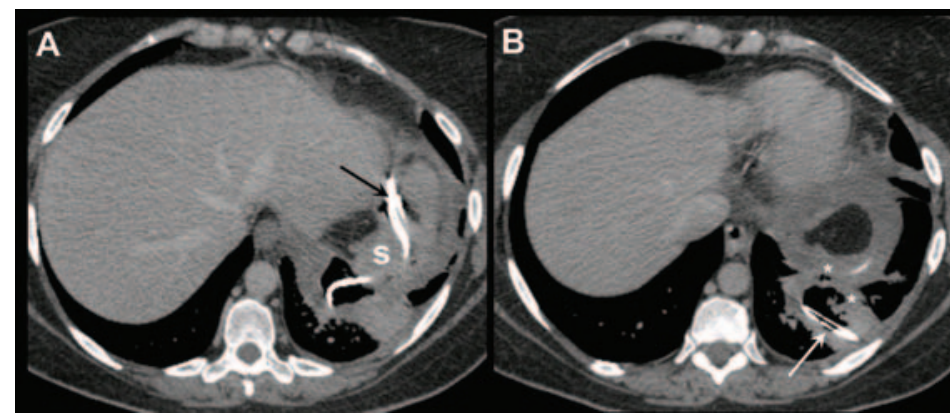

Fig. 2. Axial CT sections in a patient with GBF following laparoscopic sleeve gastrectomy. A A drain (arrow) is seen alongside the sleeve (S) and an air filled cavity lateral to it. B A higher section demonstrating the drain (arrow) crossing the diaphragm, located within a left lung consolidation, communicating with bronchi (asterisks).

Fig. 3. Water-soluble contrast swallow study (oblique view) demonstrating a contrast filled esophagus (E), sleeve (S) with a fistulous connection (arrowhead) to left bronchi (B), which are filled in a retrograde fashion.

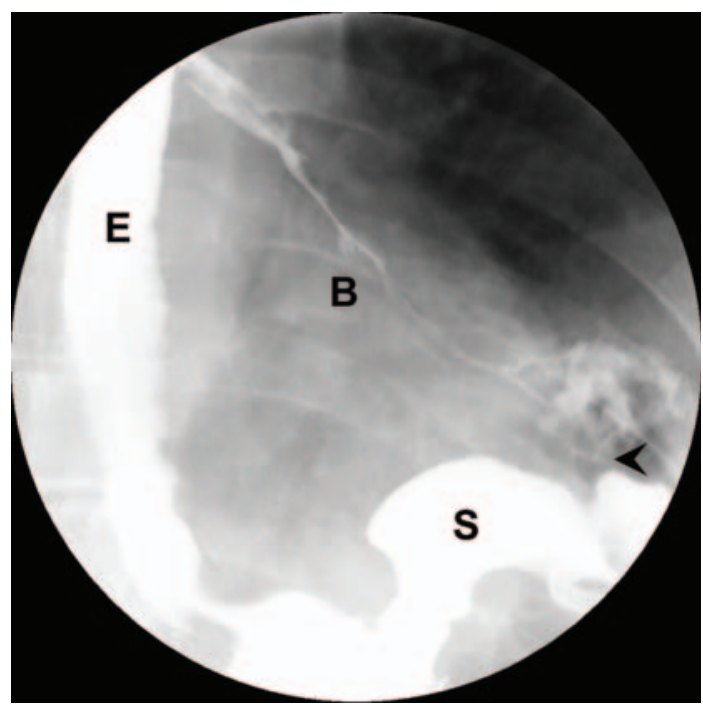


Table 2. Presentation, management and outcome

\begin{tabular}{|c|c|c|c|c|}
\hline $\begin{array}{l}\text { Number } \\
\text { of patients }\end{array}$ & $\begin{array}{l}\text { Time to } \\
\text { presentation }\end{array}$ & Clinical presentation & Management & Outcome \\
\hline 3 & 2-4 days & $\begin{array}{l}\text { immediate leak } \\
\text { exploration, washout \& } \\
\text { drainage } \\
\text { chronic leak } \\
\text { left diaphragm erosion } \\
\text { GBF }\end{array}$ & $\begin{array}{l}\text { thoracoabdominal, left } \\
\text { anterior temporal lobectomy, } \\
\text { resection + Roux-en Y } \\
\text { esophagojejunostomy } \\
\text { (2 patients) } \\
\text { drainage (1 patient) }\end{array}$ & $\begin{array}{l}\text { healed (after 2nd } \\
\text { surgery) } \\
\text { breakdown, } \\
\text { eventual colon } \\
\text { interposition, healed } \\
\text { healed ( } 45 \text { days) }\end{array}$ \\
\hline 2 & 2-4 weeks & $\begin{array}{l}\text { perisleeve collection } \\
\text { left diaphragm erosion } \\
\text { GBF }\end{array}$ & $\begin{array}{l}\text { aggressive drainage + } \\
\text { nasojejunal feeding }\end{array}$ & $\begin{array}{l}\text { healed }(6 \mathrm{~m}) \\
\text { healed }(2 \mathrm{~m})\end{array}$ \\
\hline 1 & 5 months & $\begin{array}{l}5 \text { months did well } \\
\text { left lower lobe 'pneumonia' } \\
\text { clip expectoration }\end{array}$ & $\begin{array}{l}\text { laparoscopic exploration, } \\
\text { RYGB }\end{array}$ & $\begin{array}{l}\text { healed (after 2nd } \\
\text { surgery) }\end{array}$ \\
\hline
\end{tabular}

$\mathrm{d}=$ Days; $\mathrm{wk}=$ weeks; $\mathrm{m}$ = months; $\mathrm{GBF}=$ gastrobronchial fistula; $\mathrm{RYGB}=$ Roux-en $\mathrm{Y}$ gastric bypass.

Management strategies differed among the three groups as well. Table 2 summarizes presentation, management, and outcomes in all 6 patients.

The 3 patients with a frank leak were initially treated by reoperation with washout and drainage. No primary repair was attempted due to local tissue conditions. All 3 had leaks that were found at the proximal stapler line - near the angle of His. These patients continued to develop a chronic leak. All 3 required total parenteral nutrition and multiple replacements/repositioning of drains during their long course. A left subphrenic abscess was intermittently present during this time frame as well as left pleural effusion and passive atelectasis of the left lower lobe with consolidation. Pulmonary symptoms, including shortness of breath, wheezing, chronic cough and mild hypoxemia, ensued later in the course. GBF was diagnosed at this time, 3-5 months after surgery. Two of these 3 patients required major surgical intervention through a thoraco-abdominal approach with resection of the left lower lobe of the lung, debridement of the eroded left diaphragm, completion gastrectomy, and Roux-en-Y esophagojejunostomy. One of them had a further complicated course with intrathoracic anastomotic breakdown and mediastinitis. She was operated again, with washout, resection of the anastomosis, cervical esophagostomy, and feeding jejunostomy. She recuperated slowly and underwent colonic interposition 3 months later. Complete resolution in these 2 patients occurred 8 and 11 months after initial surgery. In the 3rd, this was achieved 45 days after initial surgery, with combined abdominal and pleural drainage, and required no further intervention.

Two patients presented with abdominal pain and fever. CT scan showed a parasleeve fluid collection which was followed by CT-guided drainage. Several sessions of drain repositioning and replacement took place during a prolonged course. Patients were enterally fed via a nasojejunal tube during this period. Additional complications included upper gastrointestinal bleeding in 1 patient and deep venous thrombosis with minor pulmonary embolism in the 2nd, necessitating placement of an inferior vena cava filter. GBF was diagnosed by swallow studies and CT. Resolution was eventually achieved 4 and 6 months after surgery. 
One patient initially did well, but 5 months postoperatively developed a chronic dry cough with concomitant low-grade fever. She was found to have a left lower lobe consolidation and was treated for community-acquired pneumonia to no avail. After 4 weeks with no improvement she expectorated a surgical clip while coughing. She underwent a complete workup, including contrast swallow, gastroscopy and CT, which failed to demonstrate the fistula. She underwent laparoscopic exploration with lysis of dense adhesions near the esophagogastric junction and conversion to a Roux-en-Y gastric bypass. After surgery she did well with complete resolution of pulmonary complaints, 6 months after initial surgery.

\section{Discussion}

LSG is gaining popularity as a valid option with short- and mid-term results comparable to those of the 'gold standard' LRYGB, while carrying lower morbidity and mortality rates $[6,7]$. Through increasing utilization, better understanding of its mechanisms of action as well as technical nuances is accumulating, as are its drawbacks.

Leaks after LRYGB and LSG are uncommon and are reported to occur in $1.4-5.3 \%$ of cases $[4,14,24]$. Intraluminal pressure after LSG is high, due to the long and narrow construct with the pylorus at its end. If a leak occurs, it is difficult to treat and the course is usually prolonged. Conversely, the intraluminal pressure after LRYGB is low due to bypass of the pylorus and an anastomosis to small bowel. In this reported series, 1 of 14 patients after LRYGB and 5 of 20 patients after LSG with leak, developed GBF ( $\mathrm{p}=$ not significant (NS)).

Clinically, leaks may range from mild, such as microleaks that are suggested weeks to months after surgery as the cause of perigastric abscesses, to an abdominal catastrophe with sepsis, hemodynamic instability, multisystem organ failure, and, rarely, patient demise $[4,25,26]$.

We encountered a rare subset of these patients that developed a GBF. The clinical presentation of this complication varied among our patients and ranged from troublesome respiratory complaints, not immediately associated with the original surgery, to continued deterioration in chronically leaking patients. In all patients, chronic cough and pleuritic pain were present at, or before, the time of fistula diagnosis. Bronchoscopy, endoscopy, swallow study, and chest CT scan were performed to help establish this diagnosis. Even when contamination of the pleural cavity and pulmonary parenchyma was present, management options were not uniform. Nonoperative treatment with aggressive drainage regimens proved sufficient in 3 of 6 cases. In the other half, surgical intervention was utilized. Chronic infection of the left lower lobe of the lung necessitated partial lobectomy in 2 of these 3, performed concomitantly with diaphragm debridement, completion gastrectomy, and Roux-Y reconstruction. In the 3rd patient, conversion to a Roux-Y gastric bypass sufficed to stop the leak and allow fistula closure with subsequent lung healing.

Leak rates in patients undergoing revisional bariatric procedures are higher than those reported for primary cases $[27,28]$. Three of the 6 patients had had previous surgical intervention in the upper stomach region making this a logical risk factor for development of GBF.

Although the timing of leak diagnosis differed among our patients, GBF occurred after a prolonged course. A chronic inflammatory/infectious process under the left diaphragm seems to be the common denominator eventually deteriorating into its erosion and GBF formation. The fact that a patient has a 'controlled' leak in the gastroesophageal area, without signs of sepsis or peritonitis, should not lure the surgeon into complacency. We propose that a more aggressive approach aimed at leak management might be beneficial in preventing this dire complication. Endoscopic measures (stents, clips, fibrin glue, endosu- 
turing) with concomitant drainage ought to be used early. If these are not effective, early surgical intervention should be favorably considered.

To conclude, this rare complication of LSG and LRYGB should be suspected in the presence of pulmonary complaints, mainly incessant cough and left-sided pleuritic pain, after LSG and LRYGB. Active diagnostic measures should be employed to facilitate timely diagnosis and treatment initiation. Due to the diversity of clinical settings noted, as well as the rarity of the condition, we cannot recommend any specific treatment algorithm.

\section{Disclosure Statement}

All contributing authors declare that they have no conflicts of interest,

\section{References}

1 Baltasar A, Serra C, Perez N, Bou R, Bengochea M, Ferri L: Laparoscopic sleeve gastrectomy: a multi-purpose bariatric operation. Obes Surg 2005;15:1124-1128.

- 2 Cottam D, Qureshi FG, Mattar SG, Sharma S, Holover S, Bonanomi G, Ramanathan R, Schauer P: Laparoscopic sleeve gastrectomy as an initial weight-loss procedure for high-risk patients with morbid obesity. Surg Endosc 2006;20:859-863.

3 Nguyen NT, Root J, Zainabadi K, Sabio A, Chalifoux S, Stevens CM, Mavandadi S, Longoria M, Wilson SE: Accelerated growth of bariatric surgery with the introduction of minimally invasive surgery. Arch Surg 2005;140:1198-1202; discussion 1203.

4 Burgos AM, Braghetto I, Csendes A, Maluenda F, Korn O, Yarmuch J, Gutierrez L: Gastric leak after laparoscopic-sleeve gastrectomy for obesity. Obes Surg 2009;19:1672-1677.

5 Durak E, Inabnet WB, Schrope B, Davis D, Daud A, Milone L, Bessler M: Incidence and management of enteric leaks after gastric bypass for morbid obesity during a 10-year period. Surg Obes Relat Dis 2008;4:389-393.

- 6 Deitel M, Crosby RD, Gagner M: The First International Consensus Summit for Sleeve Gastrectomy (SG), New York City, October 25-27, 2007. Obes Surg 2008;18:487-496.

- 7 Gagner M, Deitel M, Kalberer TL, Erickson AL, Crosby RD: The Second International Consensus Summit for Sleeve Gastrectomy, March 19-21, 2009. Surg Obes Relat Dis 2009;5:476-485.

- 8 Köckerling F, Schug-Pass C: Gastroscopically controlled laparoscopic sleeve gastrectomy. Obes Facts 2009; 2(suppl 1):15-18.

9 Mognol P, Chosidow D, Marmuse JP: Laparoscopic sleeve gastrectomy as an initial bariatric operation for high-risk patients: initial results in 10 patients. Obes Surg 2005;15:1030-1033.

10 Roller JE, Provost DA: Revision of failed gastric restrictive operations to Roux-en-Y gastric bypass: impact of multiple prior bariatric operations on outcome. Obes Surg 2006;16:865-869.

11 Blachar A, Federle MP, Pealer KM, Ikramuddin S, Schauer PR: Gastrointestinal complications of laparoscopic Roux-en-Y gastric bypass surgery: clinical and imaging findings. Radiology 2002;223:625-32.

$\checkmark 12$ Hamilton EC, Sims TL, Hamilton TT, Mullican MA, Jones DB, Provost DA: Clinical predictors of leak after laparoscopic Roux-en-Y gastric bypass for morbid obesity. Surg Endosc 2003;17:679-684.

13 Marshall JS, Srivastava A, Gupta SK, Rossi TR, DeBord JR: Roux-en-Y gastric bypass leak complications. Arch Surg 2003;138:520-523; discussion 523-524.

14 Tan JT, Kariyawasam S, Wijeratne T, Chandraratna HS: Diagnosis and management of gastric leaks after laparoscopic sleeve gastrectomy for morbid obesity. Obes Surg 2010;20:403-409.

15 Gaensler EH, Jeffrey RB Jr, Noonan CD: Gastrobronchial fistula: an unusual complication of Nissen fundoplication. Gastrointest Radiol 1988;13:6-8.

16 Jha PK, Deiraniya AK, Keeling-Roberts CS and Das SR: Gastrobronchial fistula-a recent series. Interact Cardiovasc Thorac Surg 2003;2:6-8.

17 Miwa K, Takamori S, Hayashi A, Shirouzu K: Gastrobronchial fistula after esophagectomy. Eur J Cardiothorac Surg 2004;25:460.

18 Stal JM, Hanly PJ, Darling GE: Gastrobronchial fistula: an unusual complication of esophagectomy. Ann Thorac Surg 1994;58:886-887.

19 Jeganathan R, Pore N, Clements WD: Gastrobronchial fistula - a complication of splenectomy. Ulster Med J 2004;73:126-128.

-20 Campos JM, Siqueira LT, Ferraz AA, Ferraz EM: Gastrobronchial fistula after obesity surgery. J Am Coll Surg 2007;204:711.

-21 Campos JM, Siqueira LT, Meira MR, Ferraz AA, Ferraz EM, Guimaraes MJ: Gastrobronchial fistula as a rare complication of gastroplasty for obesity: a report of two cases. J Bras Pneumol 2007;33:475-479. 
-22 Chin PL: Gastrobronchial fistula as a complication of laparoscopic adjustable gastric banding. Surg Obes Relat Dis 2008;4:671-673.

-23 Garrett KA, Rosati C: Gastro-broncho-pleural fistula after laparoscopic gastric band placement. Obes Surg 2009;19:941-943.

24 Chen B, Kiriakopoulos A, Tsakayannis D, Wachtel MS, Linos D, Frezza EE: Reinforcement does not necessarily reduce the rate of staple line leaks after sleeve gastrectomy. A review of the literature and clinical experiences. Obes Surg 2009;19:166-172.

-25 Casella G, Soricelli E, Rizzello M, Trentino P, Fiocca F, Fantini A, Salvatori FM, Basso N: Nonsurgical treatment of staple line leaks after laparoscopic sleeve gastrectomy. Obes Surg 2009;19:821-826.

-26 Marquez MF, Ayza MF, Lozano RB, Morales Mdel M, Diez JM, Poujoulet RB: Gastric leak after laparoscopic sleeve gastrectomy. Obes Surg 2010;20:1306-1311.

27 Elazary R, Hazzan D, Appelbaum L, Rivkind AI, Keidar A: Feasibility of sleeve gastrectomy as a revision operation for failed silastic ring vertical gastroplasty. Obes Surg 2009;19:645-649.

28 Goitein D, Feigin A, Segal-Lieberman G, Goitein O, Papa MZ, Zippel D: Laparoscopic sleeve gastrectomy as a revisional option after gastric band failure. Surg Endosc 2011;25:2626-2630. 